\title{
Viability of a Reusable In-Space Transportation System
}

\author{
Sharon A. Jefferies. ${ }^{1}$ \\ NASA Langley Research Center, Hampton, VA, 23681 U.S.A. \\ Carey M. McCleskey ${ }^{2}$ and Brian Nufer ${ }^{3}$ \\ NASA Kennedy Space Center, FL 32899 U.S.A.
}

\begin{abstract}
Roger A. Lepsch ${ }^{4}$, Raymond G. Merrill ${ }^{5}$, David North ${ }^{6}$, John Martin ${ }^{7}$, and D. R. Komar ${ }^{8}$ NASA Langley Research Center, Hampton, VA, 23681 U.S.A.
\end{abstract}

The National Aeronautics and Space Administration (NASA) is currently developing options for an Evolvable Mars Campaign (EMC) that expands human presence from Low Earth Orbit (LEO) into the solar system and to the surface of Mars. The Hybrid in-space transportation architecture is one option being investigated within the EMC. The architecture enables return of the entire in-space propulsion stage and habitat to cis-lunar space after a round trip to Mars. This concept of operations opens the door for a fully reusable Mars transportation system from cis-lunar space to a Mars parking orbit and back.

This paper explores the reuse of in-space transportation systems, with a focus on the propulsion systems. It begins by examining why reusability should be pursued and defines reusability in space-flight context. A range of functions and enablers associated with preparing a system for reuse are identified and a vision for reusability is proposed that can be advanced and implemented as new capabilities are developed. Following this, past reusable spacecraft and servicing capabilities, as well as those currently in development are discussed. Using the Hybrid transportation architecture as an example, an assessment of the degree of reusability that can be incorporated into the architecture with current capabilities is provided and areas for development are identified that will enable greater levels of reuse in the future. Implications and implementation challenges specific to the architecture are also presented.

$\begin{array}{ll}\text { AERCam } & =\text { Autonomous Extravehicular Activity Robotic Camera } \\ A R \& D & =\text { Automated Rendezvous and Docking } \\ B L T & =\text { Ballistic Lunar Trajectory } \\ B O L & =\text { Beginning of Life } \\ E M C & =\text { Evolvable Mars Campaign } \\ E P & =\text { Electric Propulsion } \\ E S A & =\text { European Space Agency } \\ G H e & =\text { Gaseous Helium }\end{array}$

\footnotetext{
${ }^{1}$ Aerospace Engineer, Space Mission Analysis Branch, NASA Langley Research Center, mail code 462, and AIAA Senior Member.

${ }^{2}$ Technical Manager, Engineering \& Technology Directorate, NASA Kennedy Space Center, mail code NE-EM, and AIAA Senior Member.

${ }^{3}$ Aerospace Engineer, Engineering \& Technology Directorate, NASA Kennedy Space Center, mail code NE-M4.

${ }^{4}$ Aerospace Technologist, Vehicle Analysis Branch, NASA Langley Research Center, mail code 451, and AIAA Member.

${ }^{5}$ Aerospace Engineer, Space Mission Analysis Branch, NASA Langley Research Center, mail code 462, and AIAA Senior Member.

${ }^{6}$ Aerospace Engineer, Space Mission Analysis Branch, NASA Langley Research Center, mail code 462.

7 Aerospace Technologist, Vehicle Analysis Branch, NASA Langley Research Center, mail code 451, and AIAA Member.

${ }^{8}$ Aerospace Technologist, Vehicle Analysis Branch, NASA Langley Research Center, mail code 451, and AIAA Senior Member.
}

American Institute of Aeronautics and Astronautics 


$\begin{array}{ll}H P S & =\text { Hybrid Propulsion System } \\ I s p & =\text { Specific Impulse } \\ I S R U & =\text { In Situ Resource Utilization } \\ I S S & =\text { International Space Station } \\ \text { LDHEO } & =\text { Lunar-Distance High Earth Orbit } \\ L D R O & =\text { Lunar-Distance Retrograde Orbit } \\ L G A & =\text { Lunar Gravity Assist } \\ M M H & =\text { Monomethyl Hydrazine } \\ N A S A & =\text { National Aeronautics and Space Administration } \\ N T O & =\text { Nitrogen Tetroxide } \\ O M S & =\text { Orbital Maneuvering System } \\ O P F & =\text { Orbiter Processing Facility } \\ P M D & =\text { Propellant Management Device } \\ R 2 & =\text { Robonaut 2 } \\ R C S & =\text { Reaction Control System } \\ R R M & =\text { Robotic Refueling Mission } \\ S E P & =\text { Solar Electric Propulsion } \\ S S M E & =\text { Space Shuttle Main Engines } \\ S T S & =\text { Space Transportation System } \\ T L I & =\text { Trans-Lunar Injection } \\ U D M H & =\text { Unsymmetrical Di-methyl Hydrazine }\end{array}$

\section{Introduction}

$\mathrm{A}$ S humans strive to extend out into the solar system on multi-year missions far beyond low Earth orbit and make repeated visits to the same locations, the potential for incorporating reusable systems into the spaceflight architectures needs to be understood and their potential impact on exploration campaigns assessed. This paper presents a vision for reusability in spaceflight, discusses needed capabilities and challenges that must be overcome to enable increasing degrees of reusability and achieve that vision, and examines past and current attempts at reusable systems to better understand the degree of reusability that can likely be achieved in the near term. Using a general set of broadly applicable reusability functions, this paper covers an investigation of the capabilities needed to prepare systems for reuse between missions and identifies several key challenges that limit or prohibit reusability of some systems. Capability gaps and challenges provide insight into the types of investments that can enable higher degrees of reusability and promote implementation of reusable architectures.

While beginning with a general discussion on reusability, the particular focus of this paper is on the viability of incorporating reusability into the Evolvable Mars Campaign (EMC) and identifying those capabilities that would particularly benefit EMC architectures. The EMC is an ongoing NASA study investigating potential options and key decision points to enable crewed Mars missions beginning in the mid-2030s timeframe that lead to pioneering and eventual human settlement. The EMC follows a flexible strategy that leverages existing capabilities to facilitate nearterm exploration while developing a portfolio of evolving capabilities that provide specific functions to solve challenges associated with extending human presence, thus enabling more complex operations over time. One of strategic principles of the EMC is to develop multi-use, evolvable space infrastructure, minimizing unique major developments, with each mission leaving something behind to support subsequent missions. ${ }^{1}$

The EMC Hybrid architecture was investigated to assess the implications and viability of incorporating reusability into a specific EMC mission architecture. The Hybrid architecture is one of two transportation system options being assessed in 2015 under the EMC. ${ }^{2}$ The architecture is built around a hybrid propulsion system that combines both solar electric propulsion and a chemical propulsion system into a single transportation stage. The propulsion stage design is the same for both crew and cargo delivery. In a reusable instantiation of the Hybrid architecture, a small fleet of hybrid stages are produced that all return to Earth vicinity at the completion of the mission for refurbishment and resupply in preparation for the next mission. In addition to the propulsion stage, the transit habitat for the crew is also reused. ${ }^{3}$ While both propulsion and habitation modules are reused in the architecture, this paper focuses on reusability of the propulsion module. The high-level functions presented in the general discussion provide background for examining the capability needs and challenges associated with incorporating reusability into the Hybrid architecture. The degree of reusability that can reasonably be achieved with current capabilities in the Hybrid architecture is discussed and the near-term developments that can enhance or enable use of reusable systems are identified. The paper 
closes with a broader look at an approach to advance the evolution of reusability and servicing capabilities to achieve a vision of spaceflight where the systems exhibit a level of reusability akin to aircraft today.

\section{Why Reusability?}

The introduction of reusable systems into a space architecture provides opportunities not only for the EMC, but also for the future development of space. Within a campaign such as the EMC, reusable systems provide value for both in-space systems and destination systems. Incorporating reusable in-space transportation systems, such as propulsion modules and transit habitats, reduces the need to launch replacement systems, thus either reducing the number of launches or providing opportunities to launch other payloads. While both expendable and reusable architectures result in a collection of in-space assets that must be dispositioned after completion of their primary mission, there is a distinct difference between the two architectures. The hardware for disposal in expendable architectures typically includes expensive, human-rated propulsion stages and habitation modules that have robust designs capable of surviving multi-year journeys to Mars. In a reusable architecture, however, the remaining systems are primarily logistics modules and tankers that have a shorter design life and generally do not need to be human rated, and are thus less expensive to develop and produce.

Destination architectures also benefit from the ability to reuse major surface systems like habitats and pressurized rovers, particularly when crews begin to visit the same location over several missions. Reusing major systems enables the delivery of other payloads that can further expand capabilities at Mars, facilitating pioneering efforts and progression from an initial emplacement phase through a Mars surface proving ground to utilization. ${ }^{4}$ Lessons gained from early incorporation of reusability into in-space systems can be leveraged by the surface systems. Similarly, distance to Mars and lack of access to surface systems between missions present challenges to long-duration operation and reuse of surface systems, and will drive development of advanced capabilities that will facilitate reusability and servicing of in-space assets, as well.

To support a reusable EMC architecture, new systems must be introduced to resupply transportation assets and ferry new payloads to aggregate with transportation stacks. The need for, and availability of, such assets provides an initial capability that can be leveraged to build in-space servicing and manufacturing infrastructure, eventually reducing dependence on Earth-based launch of systems and materials. The initial need to refuel and resupply transportation elements provides an early opportunity for commercial and international partnerships to provide these critical services. With an initial customer base provided by the EMC, the experience gained by providers can lead to growth of servicing capabilities and the development of new technologies and capabilities that increase the degree of reusability of space systems.

Over time, repurposed assets such as retired transportation stages and emptied logistics and fuel modules can be aggregated together to form the basis of in-space supply points, which could be restocked either from Earth or with space-based resources, or they can provide the building blocks of infrastructure to facilitate servicing reusable systems between missions. They could also be broken down into resources themselves to provide feedstock for space-based manufacturing.

\section{What Does it Mean to be Reusable?}

\section{A. Defining Reusable}

For the purposes of this study and paper, reusability is:

The ability to use a system for multiple missions without the need for replacement of systems or subsystems. Ideally, only replenishment of consumable commodities (propellant and gas products, for instance) occurs between missions.

\section{B. Phases and Functions Associated With the "Reset" of Space Vehicles}

There are three stages associated with the reset period: arrival operations, space vehicle re-assembly and servicing operations, and departure operations. Within these three timeframes of activity, a variety of functions may be required depending on the achieved degree of reusability. The team has identified a list of potential reusability functions that are broadly applicable to a variety of reusable systems and architectures. As the functions required to reset a system depend on the system's purpose, operations, and associated technologies, it is neither necessary nor expected that all of these functions will be performed for any given system. This generic function list, considered for each candidate approach, is depicted in Figure 1.

American Institute of Aeronautics and Astronautics 


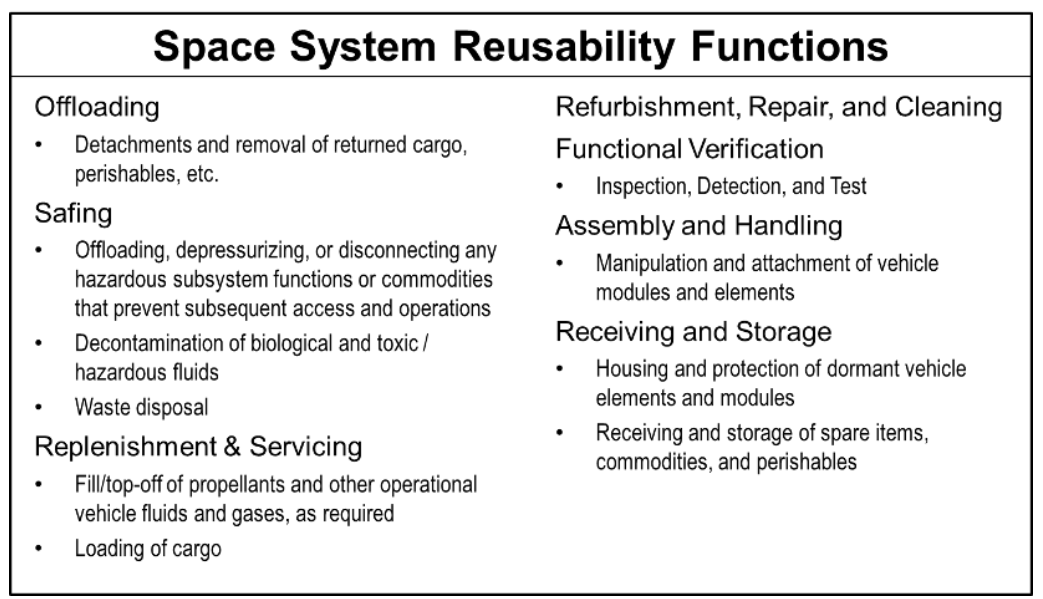

Figure 1. General functions for preparing space systems for reuse.

As stated in the Vision for Reusability detailed below, between arrival and departure a reusable system should strive for simple operations that only offload delivered crew and cargo items and take on a new load of propellants, cargo, and crew supplies. However, no matter how well the spacecraft design is planned, some degree of refurbishment, repair, and verification will be required. All of these activities must be addressed during the reset period.

\section{Vision for Reusability as an Enabler for Pioneering Operations in Space}

Envisioning space operations that pioneer healthy, sustainable growth in human activities over the long term must include greater space system reusability. The following nine-point vision provides a point of departure for organizing major technical goals and objectives for achieving the benefits of reusability-which are critical to achieving the vision for pioneering space-within the context of a campaign of pioneering journeys to Mars. The specific points emerge from insight gained during decades of previous space achievements, as well as from on-going research and analysis activity in space operations and support. It is also inspired by early investigations into innovative Mars mission campaigns intended to evolve into a state of pioneering and settlement on the surface of Mars, as well as its moons. Furthermore, it has been inspired by requests to investigate the reuse of many key elements required in pursuing such visionary campaigns.

1. Provide a simplified, very-highly automated architecture of space flight vehicles enabling minimum periodic and repetitive maintenance (airplane-like) and resultant short vehicle reset time between missions.

2. Design-in performance margins and flight hardware allowances to reduce or eliminate unplanned reset work, i.e., strive to design-out and eliminate sources of unscheduled repairs and associated tasks.

3. Strive to isolate external space vehicle support operations from dependence on specialized, unique space facilities, support systems, and equipment. At the same time, maximize use of common in situ resources and support systems. Routine, scheduled space vehicle turnaround should replenish consumables only and thus avoid vehicle reset operations becoming a time-consuming, laborintensive, infrastructure-intensive engineering effort.

4. Incorporate advanced automation and robotic capabilities that inform the operator only of the required corrective actions needed prior to the next mission. Avoid designs that lead to requirements for complex functional tests, and let the space vehicle communicate with the operator remotely during the activity in which ever domain he or she may reside. Incorporation of special engineering instrumentation will be used on specifically designated technology demonstration vehicles in space "proving ground" environments only, similar to the developmental instrumentation that was incorporated on Space Shuttle Orbiter OV-102 (Columbia). Satisfaction of proving ground certification criteria will remove the requirement on operational space vehicles.

5. Strive to validate hardware and software design complexity prior to proving ground-phase deployment and verify not only system performance, but also major reductions in space operations in the proving ground environments. Demonstrate that opportunities for "hands-on" activities are

American Institute of Aeronautics and Astronautics 
nearly eliminated. If successfully demonstrated, this will mean far fewer opportunities for human error to exist during 1,000-day-class missions and permanent space facility occupation.

6. Employ highly autonomous support system management planning in all domains-ground, inspace, and surface environments - and at the highest architectural levels achievable. Focus on automatic, on-demand scheduling of space vehicle activity, support system and equipment operation, as well as sustaining affordable and productive supply chains-extending from the Earth to a growing human presence in lunar-planetary space.

7. Promote and adopt common packaging interfaces to assure maximum flexibility, productivity, and affordability for exchange of personnel and material to and from a variety of space destinations and delivery waypoints. The most affordable and productive elements of space architectures will be blind to the cargo and personnel needs which they carry; like today's commercial transportation on the ground, on the sea, and in the air. Keep in check the impacts of payload package uniqueness so that accumulation of space system support infrastructure does not become burdensome. The accumulation of burdensome infrastructure relative to the in-space population and cargo throughput will slow productivity and add cost to the various pioneers, settlers, and their financial sponsors.

8. Incorporate a level of component commonality that allows for the potential exchange of hardware between systems in the event of component failure, while not inflicting a burdensome level of commonality that adversely affects the functional capabilities of the systems.

9. Exploration and prospecting form the foundation for pioneering. Pioneering systems are designed to grow at the destinations and are there to be used by pioneers and settlers-and not necessarily for exclusive use by scientists and engineers. The role of science and engineering during the pioneering and settlement phases will be to perform further research, expanded explorations, continuous improvement at the destinations, and technology advancement for future market-driven needs and government requirements.

It is important to note that achieving the vision of full reusability will not happen immediately given the state of current capabilities and technologies. New capabilities and technologies that facilitate reuse will need to be developed and incorporated in a manner that benefits the overall campaign. However, designing near-term systems for an achievable level of reuse and then operating those systems provides valuable lessons that, along with advancing technologies and new capabilities, will enable systems to evolve to a greater degree of reusability over time.

\section{The Importance of Retaining Functional Integrity between Uses}

Reusable systems, in practice, can exhibit large variations in time and expense between reuses. The degree of variation depends heavily on how well the functional integrity of the vehicle is retained between missions; i.e., the vehicle, its elements, and its systems drive the time and expense involved in the reset, or turnaround, activity required. What is functional integrity, however, and can it be improved by design, or through advances in technology?

Regardless of where the reset may occur, when a fully operational vehicle arrives at a location to be reset for next use, its system functions should remain intact without any disconnections, degradation, or otherwise loss of "integrity" of what had been verified and established with confidence prior to its last use.

If it can be established, through repeated use, that a vehicle retains its functional integrity without requiring replacement or repair of various components or functional paths, then there is engineering confidence in reusing the system efficiently. If however, unplanned repair and associated tasks are routinely encountered between every use, then there is likely to be a general lack of confidence, and additional time and effort will be required to re-establish functional integrity and recertify the system between missions.

While this degree of confidence may seem unreasonable in this era of infrequent Mars exploration missions, tomorrow's pioneering activity will, nevertheless, require a greater pace of activity, and thus greater confidence in system reuse. With this in mind, the proving ground concept allows for engineering improvements in a fix, fly, and fix again development approach, and will provide us the opportunity to build in the degree of system functional integrity retention required.

In more specific system engineering terms, between missions an ideal reusable space vehicle retains the following:

- Structural integrity - structural elements, assemblies, and members remain undisturbed and without component replacement. No disassembly or unacceptable conditions, such as: corrosion, erosion, punctures and dents, fractures and cracks, or loose structural connections require repair. In a robust design, many of these degradation conditions are accommodated between uses, or between extended periods of use.

American Institute of Aeronautics and Astronautics 
- Mechanical integrity - movable assemblies, such as articulating solar arrays, retain their functional integrity without component replacement or intrusive servicing and maintenance (such as calibrations, adjustments, disconnections and re-riggings, lubrications, etc.)

- $\quad$ Fluid integrity - propellant and gas commodities in the propulsion system, as well as fluids and gases in other vehicle systems (such as thermal management and environmental control hardware) remain "leak tight". No component replacements occur with fluid connections. If any of the commodity has been consumed during its flight use, then full functional integrity is retained when it is replenished through a simple external connection; i.e., without disconnecting vehicle elements, or otherwise disturbing fluid line transfer paths (tubes, hoses, flex lines, etc.), and other filtration devices, fluid vessels, and fluid system components. Doing so allows opportunities for seal disturbance, contamination, or undesired entrainment. Once a fluid line - particularly pressurized fluid lines - are disconnected, they require even more effort to re-service and to re-establish a useful operational state.

- Electrical/electronic integrity - electrical power, signal, and remote wireless connections remain undisturbed between missions. For electrical and signal path integrity to be fully retained, no connectors are de-mated and re-mated, and no intrusive calibrations or adjustments requiring disconnections for externally connected support equipment are required. Additionally, no short circuit or open circuit conditions require repair.

- Software integrity - on-board flight code remains intact and able to support the next use without the need of special vehicle system operation to verify software change functionality. Changes and upgrades to software may be accommodated, but system operation beyond otherwise normal system functional tests are not required by the software changes.

While the above list identifies an ideal that should be strived for, in reality any man-made object has limitations and could break unexpectedly, especially in the harsh environments of launch and space, which means that systems will inevitably experience a break in functional integrity. Additionally, the degree to which functional integrity can be maintained during reset is highly dependent on the system technologies and servicing capabilities available. As technologies and capabilities advance, the degree to which functional integrity is maintained will also increase. As much as possible, design decisions for reusable systems should be made to minimize the extent and impact of breaks in functional integrity, e.g., choosing to use modular, "plug-and-play"-type line replaceable units instead of selecting options requiring intrusive component-level repairs. A trade must be made to balance the mass penalties that may be incurred by selecting less mass-efficient design solutions against the time and effort saved on repair and retesting activities and the reduced risk of collateral damage provided by solutions that retain greater functional integrity.

\section{E. Degrees of Reuse}

There are many degrees of reuse that span the range from ideal single element "gas-n-go" reusability, to a single use expendable system. Partially reusable system designs come in many forms:

- Multi-element/multi-stage space transfer vehicles may have elements that are reused and elements that are expended. Elements that are recovered for reuse may require extensive teardown, or even remanufacturing. Such was the case for the ocean recovered Solid Rocket Boosters of the Space Shuttle program. Upon recovery there was very little functional integrity retained between flights.

- A vehicle element may be reused, but major assemblies or modules of the element are expended, such as an expended fuel bottle. If cleverly designed, for example a "plug-and-play" module with a minimum of connections, such systems might lose very little system functional integrity. On the other hand, if reuse is not a clear design objective, a design may be fielded with many critical functions coming apart or degrading between missions.

- A vehicle element may be fully reusable, but a number of components must be replaced based on time in service or number of cycles; such as a pressure vessel that is life-limited to a certified number of pressure cycles.

- A vehicle may have a degree of "expendability" due to frequent component failures and unplanned removals.

\section{Summary of Historic Flight and Ground Servicing of Space Vehicles}

To date, there has been limited experience with the reuse of space systems. Satellites and deep space probes have demonstrated long lifetimes in space, many operating continuously for more than 15 years without servicing. Long lifetimes have been achieved in this case through design approaches that emphasize simplicity, redundancy, and the 
use of long-life components. Once launched, these systems do not regularly experience environments, such as vibrational, detrimental to component life. There are few mechanical components to wear out, with most being passive in nature. Propulsion systems utilized are either hydrazine-based, low-thrust chemical types (typically monopropellant in low Earth orbit and bi-propellant for geosynchronous orbit and deep space), or long-duration electric types. Operations for these satellites and probes usually end when propellants have been depleted. Therefore, the reuse of space systems, at a minimum, requires servicing to replenish propellants and other consumables. At most, it may also require repair and/or replacement of failed, failing, or limited-life components.

The following sections present experiences and lessons learned from several spacecraft operations and capability development and demonstrations relevant to servicing and reusability. Additional examples beyond those included in this paper may provide additional insights relevant to reusable design.

\section{A. Space Propulsion Reusability Experience}

The Mir space station, the International Space Station, and the Space Shuttle are examples of operational designs that included reusable propulsion systems for multi-mission or extended flight operations. The transfer of propellants has been limited to the hypergolic family of propellants used in pressure-fed propulsion systems. However there have been numerous studies of potential architectures that use cryogenic propellant transfer, the most notable being those considered in the early Apollo architecture studies that looked at transfer of propellant into the Saturn V rocket while on orbit, ${ }^{5}$ as well as several recent studies investigating propellant depots. ${ }^{6,7}$ Recently there have also been several activities that are developing the new technologies needed for the next generation of reusable space propulsion systems.

\section{Progress Module, Mir (1986-2001) and International Space Station (1998-Present)}

The Soviet/Russian space station Mir used the propulsion systems on both visiting Progress resupply modules and several of the station modules for orbit station keeping. The Progress module had the capability to transfer hypergolic propellants (unsymmetrical di-methyl hydrazine [UDMH] and nitrogen tetroxide [NTO]) onto the Mir modules. The philosophy of the Mir station-keeping propulsion was to have several modules capable of orbit reboost in addition to the Progress module. The Progress M module used with Mir, and similarly the Progress M-1 module used to resupply the International Space Station (ISS), uses pressurized propellant transfer with the propellants contained in collapsible bellows to separate the propellant fluids from pressurant gases. This method is very effective and no major failures have occurred with the propellant transfer, demonstrating both the viability of pressure-fed transfer and the success of single-fault tolerant systems, however it also requires that propellant quantities in the tank remain above 50\% to extend the life of the bellows. The International Space Station has made extensive use of propellant resupply from Progress modules with over 50 successful resupply missions to ISS.

Several important lessons have been learned from Progress Module propellant transfer on both Mir and ISS. Careful purging procedures of propellant transfer lines is required to prevent frozen propellants in the lines. The highly corrosive nature of NTO can cause material degradation and requires the use of special alloys that are resistant to the rapid oxidation caused by NTO. The highly oxidizing NTO can also cause metallic oxidation "salts" to clog downstream filters. This precipitation can be exacerbated as NTO temperature decreases, which can occur during transfer if the thermal environment is not adequately managed on both sides of the transfer. During ISS refueling, thermal control in the propellant lines is exclusively via heat conduction through the resupply vehicle's structural shell. Other methods may be necessary to maintain adequate thermal control to reduce precipitation in deep space vehicles.

Both hydrazine-based fuels and NTO are highly toxic to humans. Russians perform stringent component leak tests on the ground and rely on these to justify only a gross leak check before flowing propellant when mated to client. Contamination can still occur, however, and substantial keep-out zones are required around rocket engine exhausts and vents during extra-vehicular activity to prevent EVA suit contamination being introduced into the interior of the spacecraft.

\section{Hydrazine Transfer Experiment on STS-41G}

The first demonstration of American technology for orbital refuel occurred with the Orbital Refueling System Flight Demonstration on STS-41G. During the demonstration, an EVA crew connected a hydrazine transfer line between a simulated tanker and Landsat-type propulsion system interface. Both simulated systems incorporated diaphragm tanks, which were identical to the shuttle auxiliary power unit tanks. Special tools and procedures were developed to maintain a minimum of two seals between the crew and propellant during mating to protect the crew from exposure and contamination on the spacesuits. Propellant was then transferred back and forth between the two

American Institute of Aeronautics and Astronautics 
simulated systems multiple times to demonstrate control of ullage gas recompression temperatures during refueling so that the temperatures did not reach the hydrazine decomposition temperature $\left(200^{\circ} \mathrm{F}\right) .^{8}$

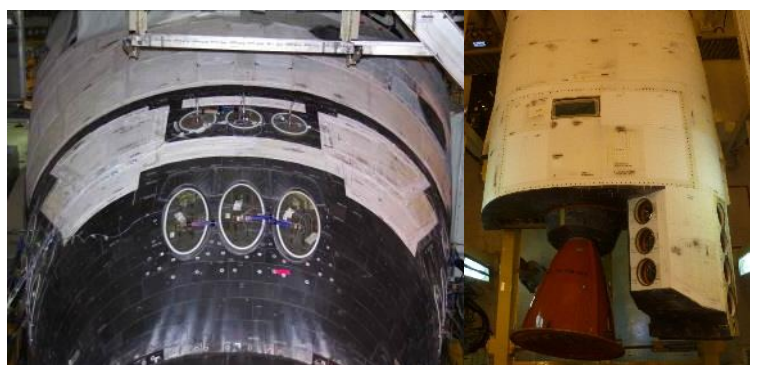

Figure 2. Space Shuttle forward RCS and aft OMS pod.
3. Space Shuttle Orbital Maneuvering System and Reaction Control System (OMS/RCS)

The Space Shuttle OMS/RCS (Figure 2) is an example of space hardware reusability and the robustness of hypergolic propellant propulsion systems. The Orbital Maneuvering Engines (OME) were designed to be capable of 100 missions, 1000 starts in space, and 15 hours of total burn time. No major failures were experienced in flight, although the system did experience component failures and propellant leaks caused by such factors as iron nitrate build-up, contamination, and filter clogging that reduced performance, imposed operational constraints, and required servicing when back on the ground. OMS/RCS incorporated redundant seals wherever possible, in series, between interior and exterior surfaces.

\section{Recent Developments for Reusable Propulsion Systems}

a) Orbital Express

In 2007 a successful in-space demonstration of autonomous servicing capabilities for a "cooperative" spacecraft and servicing vehicle designed specifically for resupply of hydrazine propellant was performed by the Orbital Express Project Astro and NextSat vehicles ${ }^{9}$ (Figure 3). The demonstration included automated rendezvous and docking (AR\&D), transfer of fluid, precise gauging of fluid transfer, and transfer of nonpropellant hardware including batteries and a computer. The propellant transfer demonstrated the use of propellant management devices (PMDs) to separate gases and liquids in the propellant tanks, proving that transfer of fuel can be accomplished without a bellows system. The propellant transfer demonstration accomplished multiple dockings and scenarios.

\section{b) NASA Technology Efforts}

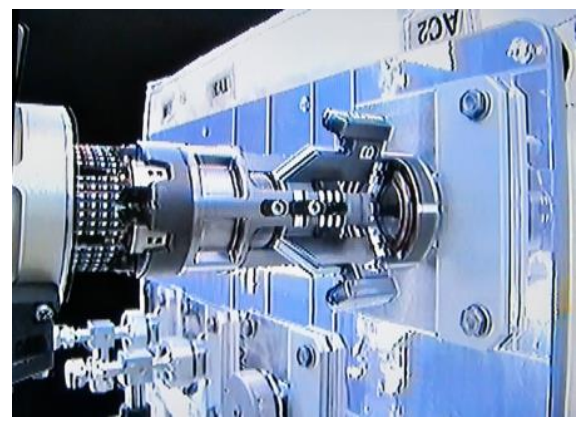

Figure 3. Multifunction tool removes plug during Robotic Refueling Mission.

NASA is currently working on key technologies that will enable automated or remote servicing of Earth satellites. The Robotic Refueling Mission (RRM) successfully performed initial experiments with in-space robotic refueling using ethanol on the International Space Station. More recently, the Remote Robotic Oxidizer Transfer Test successfully completed the ground-based demonstration of a remotely operated robotic system for servicing NTO oxidizer. A robotic arm at Kennedy Space Center was remotely controlled from Goddard Space Flight Center to make connections to a simulated client fluid transfer interface port, simulating the data and video latency of communication with an orbiting satellite. The on-orbit propellant servicing work steps were then simulated by performing interface leak checks, transferring propellant across the interface, depressurizing, and then disconnecting from the interface. NASA is also beginning early-stage development of xenon refuel technologies.

\section{B. Space Systems Servicing Experience}

Beyond the propulsion systems, the ability to service the entire spacecraft must be taken into account for reusable systems. Whereas spacecraft such as the Space Shuttle have been serviced on the ground between missions, reusable in-space systems will need to be serviced and resupplied for subsequent missions while remaining in space. In addition to the examples of capabilities supporting reusability for propulsion systems above, similar historical and current examples of servicing capabilities exist that are applicable to extending the life of the overall space system.

\section{Space Shuttle Orbiter}

With its long operational history and many lessons learned, the Space Shuttle Orbiter provides a wealth of experience with reusability that can be draw on. Although the Orbiter's operating environment, especially with launch and reentry, is more extreme than a space-based interplanetary transit vehicle will encounter, the Shuttle's reuse experience can still provide insights for future development and servicing of reusable in-space transportation systems. 
The Orbiter fleet included five vehicles that operated 135 missions over its 30-year flight program. In that time, Orbiter Discovery (OV-103) was reused the most, flying thirty-nine (39) orbital space missions from 1984 to 2011. Two Orbiters, Challenger (OV-099) and Columbia (OV-102) were tragically lost after suffering catastrophic failures. The Columbia accident in particular, which may have been mitigated were an in-space diagnosis and repair capability available, indicates the types of flexible contingency in-space servicing capabilities that future reusable vehicles may require.

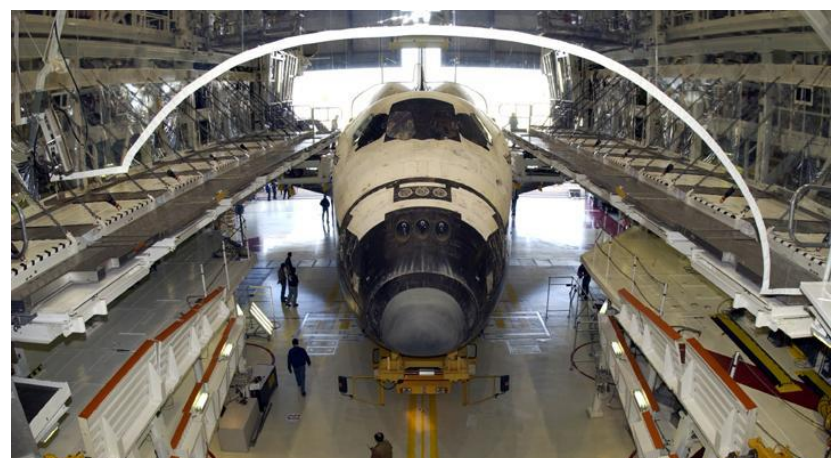

Figure 4. Space Shuttle Orbiter in the Orbiter Processing Facility

When preparing the Orbiters between missions, a typical turnaround "template" allotted 126 days from roll-in to one of three Orbiter Processing Facilities (OPFs) to lift-off (Figure 4). This consisted of 93 days for Orbiter turn-around and 7 days for Orbiter integration with the Solid Rocket Boosters and External Tank on the Mobile Launch Platform, after which the vehicle "stack" was transported to the launch pad. There, a 26-day stay was typically planned for the vehicle to connect to pad services, install the payload, arm the ordnance devices, perform launch countdown preparations and conduct the actual launch countdown to begin the flight execution ${ }^{10}$.

On a periodic basis each Orbiter would undergo a more extensive depot maintenance period, referred to as Orbiter Maintenance Depot Period, where detailed structural inspections and major component removals were required by the vehicle manufacturer and design centers. Following these periods the OPF processing time usually doubled due to the overall Orbiter functional integrity and closeout state not fully representing that of a vehicle returning directly from a space flight.

Although initially intended to achieve high flight rates, the actual achieved flight rate, which was driven by the extensive amount of work required between missions, was limited. Following the STS-51L loss of Challenger (OV099), the fleet of four Orbiters (Columbia, Discovery, Atlantis, and Endeavour) reached a stable flight rate average of 7 to 8 flights per year during the 1990s, prior to International Space Station (ISS) construction period. During the ISS construction phase, and after re-establishing "routine" operations following the loss of OV-102 Columbia, a flight rate of about 5 per year was demonstrated with the three-Orbiter fleet.

Ground turnaround operations were based on requirements defined in an Operations and Maintenance Requirements and Specifications Document that were managed and controlled by the appropriate design agency (Rockwell International and NASA JSC for the Orbiter, for example). These requirements were then satisfied through execution of a series of planned procedures that were scheduled according to the configuration of the previous mission and the needs of the up-coming mission. Depending on the nature and design of the systems involved, the planned ground turnaround procedures were executed either remotely from the Launch Control Center through automated software procedures, onboard from the Orbiter's flight deck and on-board control screens, or onsite in the OPF at one of three maintenance stations within the facility (forward, mid-body, and aft shops). Many procedures required a combination of both locally and remotely executed tasks.

Typical tasks in the OPF included tile repair; payload bay reconfiguration; visual inspections; cleaning; component/system functional testing; calibrations; and local fluid and gas servicing equipment operation. In addition to the OPF, off-line supporting repair and maintenance facilities, equipment, and services were needed, such as for the Forward RCS and aft OMS/RCS pods at the Hypergolic Maintenance Facility, an "engine shop" for the Space Shuttle Main Engines (SSMEs), and a "tile shop" for fabrication of repair of thermal protection system components.

Each SSME was routinely removed, which required intrusive operations into the aft propulsion structure, establishing access platforms and installing drag-on equipment, oxygen monitoring and lighting for personnel, as well as the removal operations itself. This drove added "closeout" work to reconfigure disconnected and disassembled components and subassemblies prior to reflight. Any work requiring component removal and replacement was typically invasive and not at all achievable during flight. Any vehicle intentionally designed to have components replaced in space will need an extensive amount of detailed engineering and thought to simplify servicing operations, maximize retention of functional integrity, and reduce the risk of collateral damage.

American Institute of Aeronautics and Astronautics 
Despite its operational robustness, the Space Shuttle OMS was never designed as an in-space-serviceable system. Although minor troubleshooting could be performed on orbit, such as the removal of the gap filler on STS-114, more serious issues had to be addressed during ground servicing. OMS/RCS propellant servicing was a complex and highly

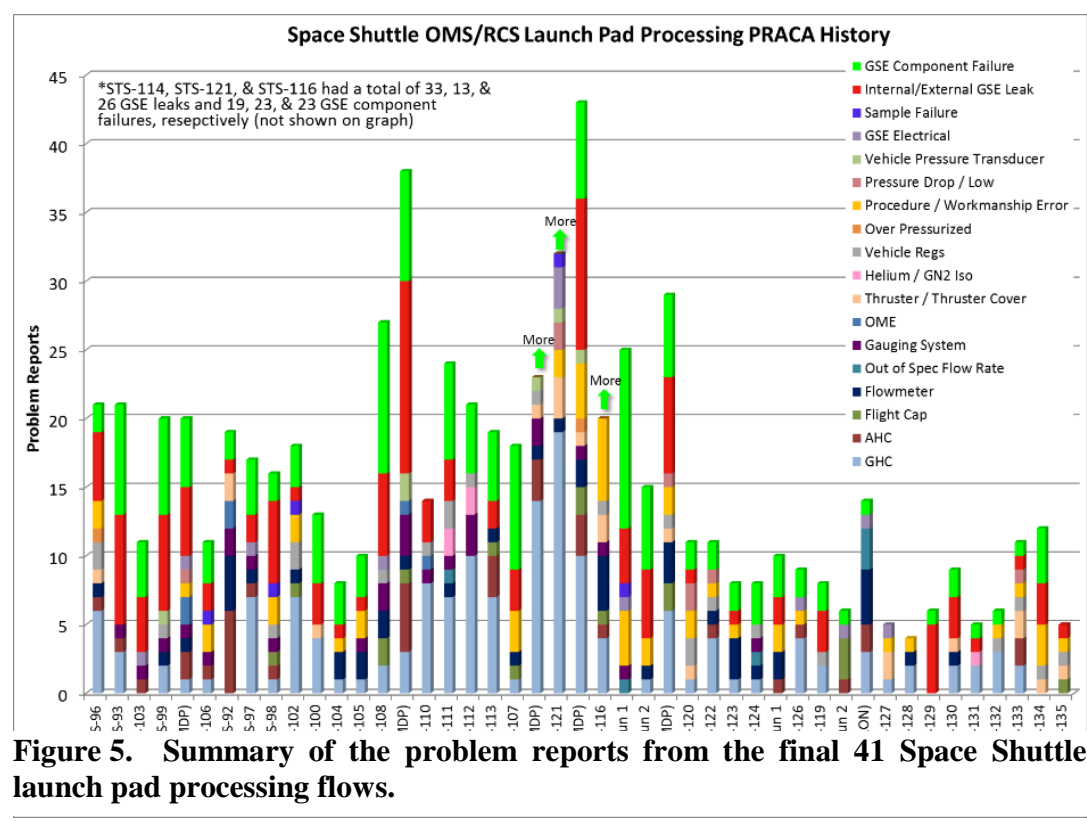

integrated task that typically occurred about two to six weeks before launch at the launch pad. It took about six to ten days to prepare the ground support equipment and orbiter for servicing, and the timeline for OMS/RCS servicing operations was on average about 30 hours in length. Over the final 41 processing flows, there was never a flow in which there were no problems related to the flight OMS/RCS or servicing GSE as a result of the system complexity, harsh corrosive environment, and toxicity or corrosiveness of the propellants (Figure 5). These servicing challenges could be magnified for inspace servicing operations unless efforts to address the problems are consciously considered in the system design phase.

The external thermal protection systems were also a major source of work, requiring not only tile repair, but rework of thermal barriers for outer mold line penetrations and various mechanically operated doors, hatches, and probes. Also included in this type of work were fabrication, repair and re-installation of payload bay liners.

The reconfiguration of the payload from the previous mission to the next mission also entailed a great deal of customized work. Work involved removal of any returned cargo, disassembly and relocation of support structure, relocation of electrical services, payload installation, thermal protection closeout, and so forth. The crew cabin accommodations and airlock/hatch arrangement were also at times reconfigured.

Beyond the planned work and the SSME, tile, and payload reconfiguration operations, a great deal of unplanned troubleshooting and repair work would arise from functional failures detected during both flight and ground operation, as well as through the required inspection procedures. On average, over 100 Line Replaceable Units - excluding SSMEs, tile, and payload cargo items - would require replacement each turnaround. Replacements resulted from: a failure detected during flight operation ( 10\% of replacements); failure detected during a planned functional verification, or replaced as a known life-limited item ( $\sim 40 \%$ of replacements); or, failures detected during the normal course of conducted ground operations (50\% of replacements), such as an Orbiter avionics box failing during a payload test.

Shuttle turnaround operations were analyzed across eight flights that launched in 1997. A summary of direct work volume concentrations is included in the pie charts of Figure $6 .{ }^{11}$ The chart does not include indirect work activity, such as offline engine repair. Also note that "processing support" work included work required in support of other operations. For instance, manning of avionics stations in support of propulsion system checkout, or hydraulic operations to move a surface for tile repair.

American Institute of Aeronautics and Astronautics 

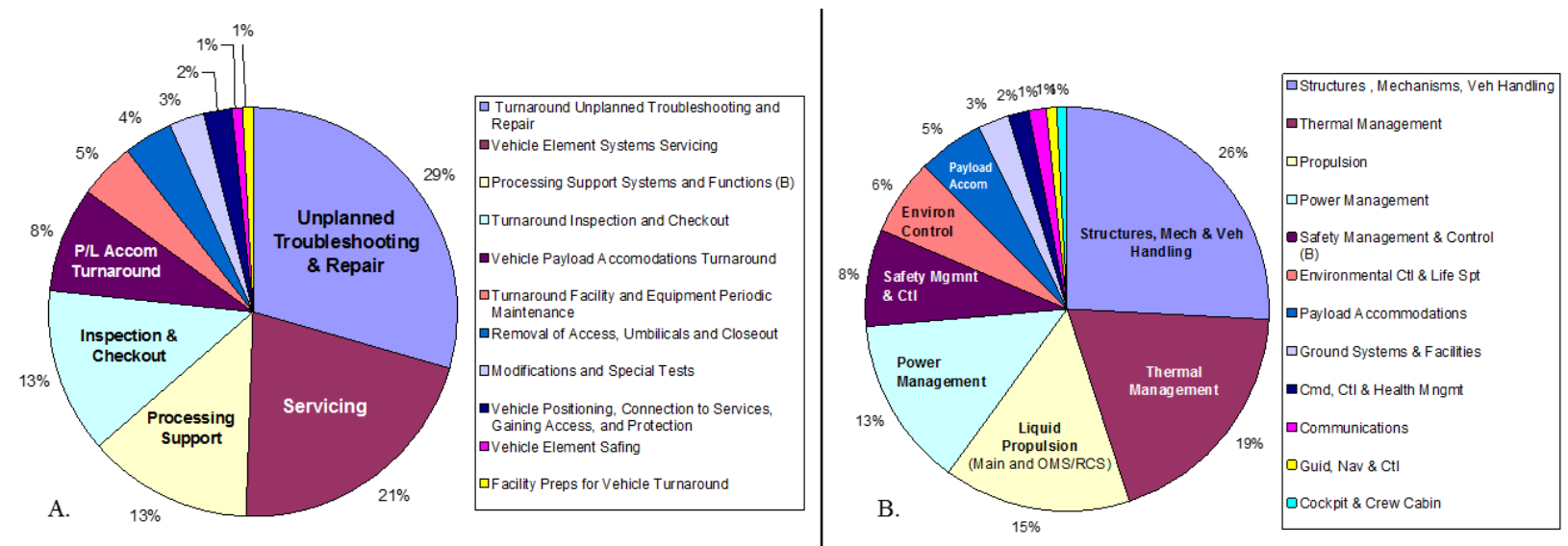

Figure 6. Space Shuttle Orbiter turnaround work volume concentrations by A) general function and B) systems variety.

As can be seen, the Space Shuttle Orbiters required an extensive amount of tear down and effort in order be readied for the next mission, which will not be a viable solution for in-space reusable assets. It is important to note, however, that a significant portion of the work is specific to the Orbiter's systems and operating environment, such as the thermal system, which had to protect the vehicle during reentry, and the SSME's, which had to push the Space Shuttle through launch. Additionally, the pre-launch environment, launch loads, and reentry stresses repeatedly experienced by the Orbiter far exceed that which an in-space transportation systems will encounter, therefor servicing requirements for in-space systems should also not be as extensive for the Space Shuttle. Despite these differences, there is still value in the many lessons learned from the years of Space Shuttle servicing and operation, which can be used to inform future vehicle designs and operations in an effort to drive less complex, more readily serviceable systems.

\section{Robotic Systems}

Progress has being made with autonomous and teleoperated in-space robotic assets that can perform servicing and maintenance tasks needed for reusability. Several robotic arms have been utilized on the Space Shuttle Orbiter and on ISS that have or are currently demonstrating functionality to maneuver spacecraft, manipulate components, and position and stabilize crew, including the Special Purpose Dexterous Manipulator, also known as Dextre, that was used to manipulate the RRM Module during the RRM (Figure 7). Small robots such as NASA's Autonomous Extravehicular Activity Robotic Camera (AERCam) ${ }^{12}$, a free-flying platform capable of obtaining difficult to reach camera views that was demonstrated on STS-87, provide utility to support in-space inspection and recertification. Since 2011, the humanoid robot, Robonaut 2 (R2), has been

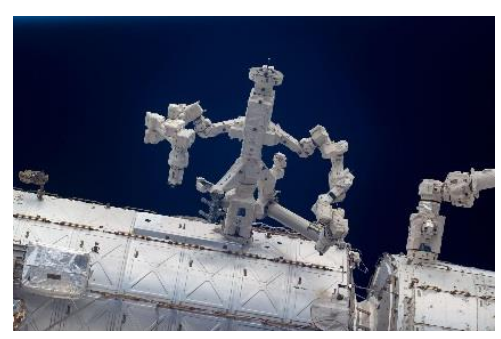

Figure 7. The Special Purpose Dexterous Manipulator on ISS. aboard the ISS demonstrating tasks that support crew inside the station ${ }^{13}$. R2 can be operated from Earth by ground controllers or by an astronaut on station through teleoperation. Future upgrades will enable demonstration and testing of extravehicular tasks. Both intra- and extravehicular activities are similar to those anticipated for facilitating vehicle reusability. 


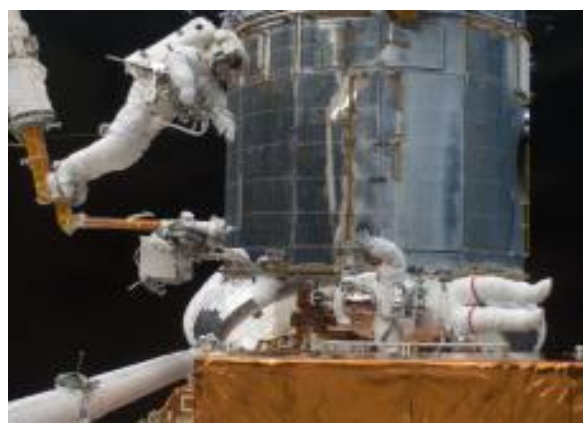

Figure 8. Crew perform maintenance during Hubble servicing mission.

\section{Hubble Space Telescope}

NASA has gained experience both in the design of a serviceable spacecraft and in successfully servicing spacecraft through the Hubble servicing missions. The Hubble Space Telescope was intentionally designed to accommodate modular upgrades and regular, periodic, servicing by astronaut crews. ${ }^{14}$ Instruments and components were modular and designed to be replaced as a unit, used standardized fittings, and were accessible to extravehicular astronauts. In addition to the servicing missions, the Hubble also incorporated redundancy and safe mode systems to facilitate reaching its 15-year planned lifetime. The servicing missions addressed three scenarios: technological upgrades, planned replacement of degraded components, and unplanned replacement due to equipment failure or malfunction (Figure 8). Although the Hubble design attempted to plan ahead to address part replacements, there were several items that had to be removed and replaced that were not originally meant to be replaced. These required the fabrication of special tools and very intricate extravehicular operations.

\section{3-D Printing Technology}

3-D printing is an emergent technology that is beginning to see use in Earth-based manufacturing of spacecraft parts and developing capabilities for in-space manufacturing. NASA and ESA have both tested ground-manufactured engine components such as thrust chambers and nozzles, and commercial companies have successfully incorporated 3-D printed parts into flown launch vehicles and satellites. Initial experiments of 3-D printing hardware on the ISS have demonstrated the ability to successfully print low-temperature plastic parts in a micro-gravity environment.

\section{Considerations Impacting Reusability}

Several factors affect the reusability of a system and the feasibility of incorporating reusability in to a mission architecture. This section will address system design factors associated with reusability, design considerations and constraints that tend to create operational tasks, and mission considerations. NASA is conducting early investigations into cis-lunar proving ground test and demonstration environments to discover when, and in what subsystem functions, the reset requirements will tend to accumulate. With such experience, developers can make informed design decisions to increase the degree of reusability of operational systems and more effectively incorporate highly autonomous systems, algorithms, and procedures to expedite the turnaround of Mars campaign elements.

\section{A. Design Factors}

Certain factors impact the capabilities needed by a reusable system beyond the types of reset activities the vehicle will encounter. As has been previously discussed, designs for reusable systems should strive to retain functional integrity to the greatest feasible extent during vehicle turnaround, within the constraints of available technologies and added system mass. Additionally, the desired system lifetime, location where the reset will occur, and the need to access subsystems and components that need servicing influence design decisions and affect the degree of reusability that can reasonably be achieved.

\section{Desired System Lifetime}

System lifetime, or number of mission cycles, is a determining factor for many system design decisions ranging from material selection and technology choices to subsystem replacement strategy. A higher degree of reuse that comes closer to the Vision outlined above may be more achievable over shorter durations and fewer cycles. There are multiple options for extending the life of a system so that it can operate for longer periods before retirement or overhaul. The primary options include designing built-in fault tolerance, planned routine replacement, or development of new or improved components with longer life.

Some degree of fault tolerance is typically designed into any spacecraft. Fault tolerance may incorporate redundancy, replication, and diversity to ensure a system will continue operating after a component failure. Redundancy involves designing in similar or dissimilar redundancy and having the subsystems pre-integrated into the vehicle from the outset. Units can either be cycled to share the operating time, or else the redundant units are kept dormant until the primary unit fails. Replication is similar to redundancy in that multiple identical units are installed in the system, but the units all run concurrently in parallel and performance of the units is checked against one another

American Institute of Aeronautics and Astronautics 
for fault detection. Diversity incorporates dissimilar systems providing the same capabilities in the same manner as replication. These methods are useful for components that are not expected to undergo significant technological development over the life of the vehicle. Some level of fault tolerance may be required for critical systems, and for those subsystems or components that will be inaccessible. Different degrees of fault tolerance are designed into a system depending on the system's criticality. The degree of fault tolerance that can be built into a system depends on the mass and volume associated with installing the additional components and the overall planned lifetime of the system.

The mass of carrying multiple units, and associated logistics burdens, would be carried throughout the entire lifetime of the vehicle. Failed units would not nominally need to be replaced unless the failure was early, unexpected and potentially indicative of a larger systemic problem. For shorter durations or fewer number of use-cycles, it may be feasible to design in enough fault tolerance to satisfy the needs of the entire planned system lifetime without having to make repairs. As lifetime durations extend, the number of units necessary to make a fully-fault tolerant subsystem (i.e., one for which you would not repair system faults or replace failed units) would grow to exceed reasonable mass and volume limits. Additionally, the accumulation of parts in the overall design may increase the odds of part failures between flights if overall design durability and life objectives are not also increased. Therefore, another approach, such as planned replacement or increased design life and quality, will be necessary. A process for developing and balancing safety, reliability, and maintainability requirements is available to mathematically explore these relationships. ${ }^{15}$

Planned routine replacement involves scheduled replacement of units based on expected mean time between failures, much like automobile maintenance today. An appropriate number of spares would need to be kept onboard to cover the routine replacements and any contingencies for the mission, and a new stock of spares would be delivered on the resupply between uses. Therefore the mass of the units carried is reduced compared to a fully redundant option. Any item that will be routinely replaced, however, should be readily accessible, which will impact the vehicle design. Routinely replaced elements that are not accessible will require more time to effect repairs, and getting to them may disrupt or damage other systems. Planned routine replacement is particularly useful for items that undergo potentially rapid technological advances, for example computers and avionics, where it may be advantageous to be able to incorporate upgrades from mission to mission as they become available.

Repeated system development cycles, whether re-engineering existing components or developing new technologies, may be the only feasible means of achieving the required lifetimes, particularly as system lifetimes extend from years to decades. An example is materials that cannot be readily replaced, such as embedded valves and seals in engines and fuel lines. Even with fault tolerance and planned replacement, components and subsystems will benefit from these development cycles to increase their lifetime and reliability. In some cases, longer subsystem/component lifetimes are necessary to enable a fault tolerant or planned replacement strategy (e.g., when you would otherwise have to carry too many spares to achieve the desired lifetime). The cost, schedule, and risk associated with an additional development cycle must be weighed against and the expected benefits to determine whether the overall improvement to the vehicle (e.g., reduction in mass carried) exceeds the cost, time required, and risk associated with the development. If not, then an alternative design solution may be necessary.

\section{System Reset Location}

A system near Earth is easier to reset than a system at Mars because of proximity. Time delays are shorter, which makes communication with a servicing crew and ground operation of robotic assets more effective and allows for a more rapid response during contingencies. Reduced transit times, days vs. months or years, enable the ability to more quickly deliver replacement parts, and potentially send a servicing crew, if an unexpected failure occurs. Furthermore, resupply will be easier the nearer the system is to the origin of the resupply, and currently all resupply is provided from Earth. Systems that must be reset on Mars may require different design decisions and incorporate different lifetime strategies than systems that return to Earth for reset. Systems being reset at Mars will also benefit from capabilities that come closer to achieving the ideal Vision for Reusability outlined above. As the capabilities for acquiring resupply from in-situ sources and manufacturing parts in space are developed, the location of the system during reset will become less of a factor.

\section{Accessibility}

Subsystems and components that will need routine servicing or repair will benefit by being readily accessible. This factor influences design decisions such as configuration and layout, and may also impact selection of the subsystems or components. Providing accessibility to components can also reduce or eliminate to need to remove other components and disturb the functional integrity of neighboring subsystems.

American Institute of Aeronautics and Astronautics 
Standardized fittings can facilitate part replacement when necessary. In addition to making components accessible, any system that will require extravehicular servicing should incorporate design features such as hand holds and foot restraints for crew, as well as power and data receptacles for robotic assets.

\section{B. Controlling Operational Tasks}

Each task created takes time to perform (whether in the critical path or not), labor (if not performed in a fully autonomous manner), material, and equipment. This resulting volume of work and accumulation of support systems and equipment can be controlled by understanding required, or planned, tasks needed by the system; and also by considering how unplanned troubleshooting and repair tasks can be controlled.

\section{Controlling Planned Work during Spacecraft Resets in Space-By Design}

Superimposing a very high-level vehicle-system breakdown with a similarly high-level breakdown of generic functions can give the conceptual designer a sense of what tasks may or may not be required to reset the vehicle and its systems. These can then be explored in more detail to see how much of the accumulation of these tasks can be reduced and controlled by design. The key, of course, is a simple design that avoids as many generic functions as practicable while retaining the performance required of the mission (Vision Point \#1). Once analyzed, the tasks that remain can be sequenced with probabilities for time and resource levels estimated to accomplish each planned task.

\section{Controlling Unplanned Work during Spacecraft Resets in Space-By Design}

One generic function included in Figure 1 is unplanned repair. Vision Point \#2 stresses designing to reduce or eliminate unplanned reset work. Time and resources may be allocated for repair, but the level of repair is largely unknown until the system is fielded-preferably during dedicated full-scale test and demonstration programs replicating the pace envisioned and the infrastructure to be drawn upon.

Specific repair tasks can be difficult to accommodate, let alone predict, in a planned routine. The reason for this can be seen by breaking down a typical repair activity on a space vehicle element:

- Identify and troubleshoot a functional failure

- Safe or configure the area to be accessed and gain access (either to further troubleshoot, or to get to the problem component(s); this will likely destroy the integrity of many functions just to perform these tasks

- Remove and replace the failed component

- Reconnect any disconnected components

- Retest the function prior to closeout

- Closeout the repaired area which was intruded into

- Final functional check-out to verify repair

If the component is accessible, a simple mold line panel, or otherwise externally accessible component will negate or highly alleviate the work involved with these sub-functions. However, if surrounded by many components and obstructions, the work involved can cause a lot of collateral removals, and possibility of damage, resulting in even more unplanned activity.

For unplanned troubleshooting and repair tasks, not only are there probabilities associated with task times and resource levels, there are probabilities of whether these operations occur at all, and to what degree. For this reason, mature commercial and military transportation systems include "type" certifications for the operational readiness of the system to determine this very effect. While flight-by-flight certifications (provided by flight readiness reviews) were conducted for each flight, this one-time "type" certification was never considered appropriate for the reusable Space Shuttle. ${ }^{16}$ This distinction is similar to the difference in turnaround requirements between experimental aircraft and commercial jetliners. Again, the space flight proving ground approach could address continuous improvement of system dependability and dramatically reduce unplanned troubleshooting and repair work by design, thus providing improved operational readiness for effective, affordable, dependable Mars campaigns (Vision Point \#5). Note that even though even though unplanned events may be significantly reduced, the unforgiving nature of the space environment means that unforeseen contingencies will likely never be completely eliminated, and vigilance will need to be maintained to detect unanticipated failures.

\section{Mission Factors}

Mission factors affecting reusability typically fall into two categories: time and workforce. The decision to incorporate reusability must consider the time required to refurbish and restock the system between uses against the 
time available, which is governed by the arrival and departure windows of the preceding and upcoming missions. Even for low delta- $\mathrm{V}$ missions to Mars where the alignment dictates a cadence of no more than one mission use for every two Mars transit opportunities, long transits between arrival orbit and cis-lunar servicing orbit limit available reset time. Time available is also dependent on the timely arrival of the resupply and servicing flights, which is influenced by the available trajectory options and propulsion system decisions. Trajectory decisions balance on the amount of time it takes for the servicing vehicles to reach the transportation stack and the delta-v required for the trajectory. If a solution is not found that fits in both the time and delta-V budgets, then resupply from Earth may not be possible and another alternative will be necessary.

Available workforce for performing reset activities includes both human crew and robotic assets. A dependence on one or the other may limit the types of reset activities that can be performed. Human crews may be able to perform tasks more quickly, but they will need logistics support during the servicing mission and they will have a limited amount of time available. Additionally, some tasks, such as refuel of hazardous propellants or maintenance in the vicinity of the large solar arrays, may be deemed unacceptably risky for humans to perform when weighed against the criticality of the task for successful completion of the mission, particularly if other, non-human assets can be provided that can perform the task. In the past there have been times, such as with the human-performed repair of an ISS solar array, where the criticality of the task made the level of risk to the crew acceptable ${ }^{17}$.

Robotic assets may have more available time if they reside with the reusable system, but they may be more limited in the types of tasks they can perform. For remote reusable systems, however, such as those on the surface of Mars, robotic assets may be the only way to maintain and prepare systems for the next mission. It is highly likely that both on-site and remote crews will have to be looked upon more extensively to effectively complete the necessary reset operations. A balance needs to be struck to efficiently perform all the necessary reset tasks in the time available to ensure the vehicle is ready for the next missions. Workforce limitations can also be mitigated by incorporating greater levels of autonomy, which is consistent with Vision Point \#4.

\section{Capability Needs and Challenges with Reusability}

\section{Architectural Need and Reusable System Goals}

The goal for incorporating reusability into an architecture is to produce highly affordable and productive spacebased vehicles and support elements that are needed for achieving the end vision of an Evolvable Mars Campaign. Reusable system goals involve responsive operations with simplified in-space turnaround times (reset time) for the elements involved. Additionally, safe flight operations are mandatory, and hardware and software reset processes must be dependably repeatable with predictable turnaround times (Vision Point \#5). Flexible configurations are also needed with an optimum degree of commonality to effectively conduct the wide array of EMC missions and affordably acquire the elements. Commonality and higher element utilization will not only demand reusability as the capability evolves and is sustained, but has the potential of lowering production unit costs, recurring ground and mission operations costs, and fixed logistical support costs by avoiding custom one-off designs for commonly performed functions.

\section{Reusable System Challenges}

There are challenges associated with achieving these reusability goals, but all can be overcome with appropriate engineering attention, technical innovation, and the patience to prove out reusability in the proving ground of nearEarth and cis-lunar space environments.

First there is the challenge of creating simplified reusable vehicles with little or no propulsive element staging, and thus, little or no resulting vehicle re-assembly and integration between missions. Simplified elements with a low number of subsystems are required to reduce design and development work for mission and support systems, as well as the recurring assembly, servicing, checkout, and repair activities. The simpler the element design, the easier it is to build and operate. The subsystems of these elements must be designed carefully to avoid — by design — unintentional or intentional disconnections or disassembly to avoid an over-accumulation of reset tasks. A balance must be maintained between the simplicity of the design and additional risk introduced by oversimplifying the design to the point where sufficient redundancy is lost or essential functionality is not adequately provided. System degradation between missions and the reuse life of the vehicle must also take into account system degradation in the various environments the system will encounter-including the reset environment itself.

Addressing the challenge of simplification should also result in another required characteristic of highly reusable, operable systems; that is a very low number of element servicing interfaces to avoid an over-accumulation of support service hookups and the resulting footprint residing at the aggregation points and destinations. Infrastructure that improves productivity is desired. Added infrastructure that does not improve the pace of EMC operations will become

American Institute of Aeronautics and Astronautics 
a burden. Therefore, greater automation and reduced need for manual assembly, servicing, and repair will also be a desired capability for a more responsive and repeatable reset process.

Propulsion systems designed with high operating margins are needed to avoid inspections, teardowns, and replacements that result from a lack of engineering confidence in the hardware. Reusable elements that design in high operating margins are far more likely to retain full functional integrity between mission cycles and demonstrate with confidence that they can be reused without such intrusive activity. A challenge will be in achieving sufficiently high margins without incurring excessive element mass and cost increases and unacceptable architecture performance impacts.

Reusability can be enabled if flight elements are easily evolvable to ISRU dependence for refueling and resupply. This will also help add inherent design and operating margin to the various elements and the robustness of the campaigns.

\section{Technical Capabilities}

The EMC concepts of hybrid chemical/electric propulsion systems ${ }^{18}$ will require large-scale, high kilowatt power systems, which in turn require highly capable thermal management and control systems. Creating highly dependable, simple to service thermal control systems, which at these scales tend to have many active components and serviceable fluid commodities, is a needed capability for reusable in-space designs. Reusable, long-life solar arrays will also be needed, along with dependable high power electric thrusters. The low power thrusters have demonstrated thousands of hours of service life. These desirable reusability characteristics need to be demonstrated at the scale of humancapable propulsion systems.

Whereas in-space transfer of hypergolic propellants has been performed on a small scale, the high-volume, inspace transfer of a variety of both chemical and EP propellants are needed capabilities that have yet to be fully developed. Along with the technical performance characteristics of the system in operation, the availability of EP propellant, in particular, should be followed through the supply chain throughout its life cycle. For example, the amount of propellant needed in the supply chain rarely equals exactly that consumed during flight. The amount required during development, qualification and acceptance testing, and demonstration flight tests must be taken into account, and losses in the support systems and the many equipment transfers should also be considered. Highly autonomous propellant management systems are also needed to demonstrate easy-to-operate/automate in situ support elements of the EMC architecture.

\section{Reusability in the Hybrid Propulsion System Architecture}

\section{A. Architecture Description}

An investigation into the implications and feasibility of incorporating reusability into a specific mission architecture was performed on the EMC Hybrid architecture. The Hybrid was chosen because the mission design enables round trip missions to Mars and recapture back at Earth without staging any part of the transportation system. Additionally, all fuel required for the mission is onboard at Earth departure, allowing for all reset operations to be performed in cis-lunar space. ${ }^{19}$ The in-space transportation system for the Hybrid architecture combines chemical and solar electric propulsion systems into a single stage. The GRC COMPASS team, in collaboration with the authors, developed concepts for both a Hybrid propulsion stage (HPS) (Figure 9) and a refueling tanker to resupply the HPS with fuel (see Section VII.B.1.a below). The concept is based off currently available components where possible and is limited to supporting three missions to Mars, with element lifetime of approximately 15 years.

The chemical propulsion system for this concept uses hypergolic bipropellant (MMH and NTO) and leverages existing technologies rated for the demands of 3 round trip Mars missions, including the Aerojet R-42 engine. The SEP component of the concept uses components developed and tested on the Asteroid Redirect Mission SEP stage..$^{20}$ In order to provide

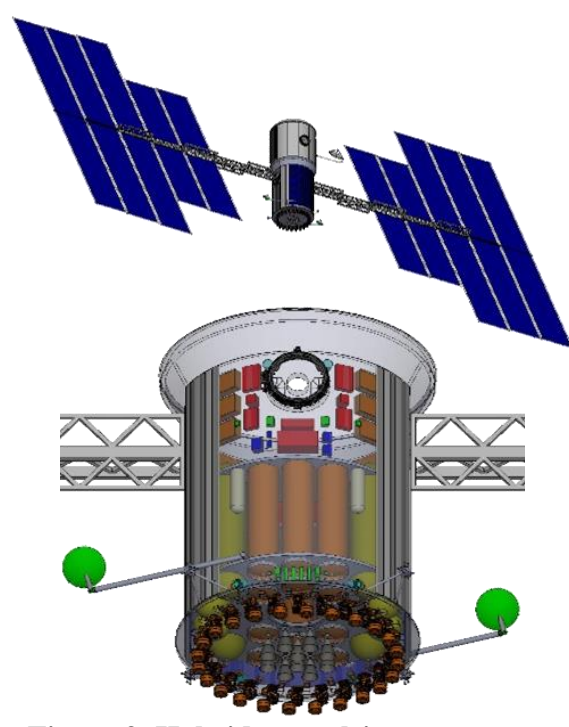

Figure 9. Hybrid propulsion stage enough power for the vehicle end of life at Mars distance, the solar arrays are sized to provide $435 \mathrm{kWe}$ of power at $1 \mathrm{AU}$ at beginning of life (BOL). The spacecraft is still power limited even at this power level and would benefit from more array power. The electric propulsion system is made up of 24 strings including power processing units, xenon feed systems, and magnetically shielded hall thrusters that utilize a combined 
input power of approximately $318 \mathrm{~kW}$ and can operate in high Isp ( 3000s) or low Isp ( 2000s) modes. In the architecture, the HPS concept is used for both crew and cargo missions to minimize the number of unique elements. It is sized to deliver the EMC in-space habitat concept (41.5 t) round trip, and is able to deliver the EMC 18t payload lander concept (43.6 t) and return without a payload for less than the maximum propellant load. ${ }^{21,22}$

The HPS is deployed for use via an SLS block 2 to trans-lunar injection (TLI) to a lunar gravity assist (LGA) in order to minimize array degradation due to the Van Allen Belts. From there the HPS flies on a ballistic lunar transfer (BLT) to cis-lunar space (lunar distant retrograde orbit, LDRO) in order to minimize fuel requirements. ${ }^{23}$ The HPS is then refueled in cis-lunar space (LDRO) and prepared for its mission. The propellant required for the mission is supplied by a tanker module that is launched to rendezvous with the HPS in cis-lunar space. It carries a combination of hypergolic bi-propellant and xenon. The specific ratio of each fuel type is dependent on the HPS concept and the maximum payload is limited by the SLS block 2 throw capability. The current concept can delivery up to $10 \mathrm{t}$ hypergolic bi-propellant and $35 \mathrm{t}$ of xenon in a combination that does not exceed $39 \mathrm{t}$.

After checkout, fueling, and logistics supply, the HPS and payload transfer to a lunar-distance high Earth orbit (LDHEO) for rendezvous with the crew prior to departure. Cargo-only missions do not require return to a specific LDHEO. Rather, they can depart with a BLT, which increases their energy with respect to the Moon, and then perform a series of LGA that propel the spacecraft to a $\mathrm{C} 3=2 \mathrm{~km}^{2} / \mathrm{s}^{2}$ with the correct declination for a transit to Mars. To complete a mission to Mars, cargo transit stages return to a LDRO after delivering cargo to the Mars sphere of influence, crew transit stages return to LDHEO to rendezvous with Orion and drop off returning crew prior to transiting to LDRO for resupply and refurbishment.

A high-level depiction of the reset operations phase is depicted in Figure 10. With currently assessed trajectories, the time available for the reset phase ranges from $~ 415-600$ days, although this also includes transit time between LDHEO and LDRO, which could account for as much as 400 of those available days.

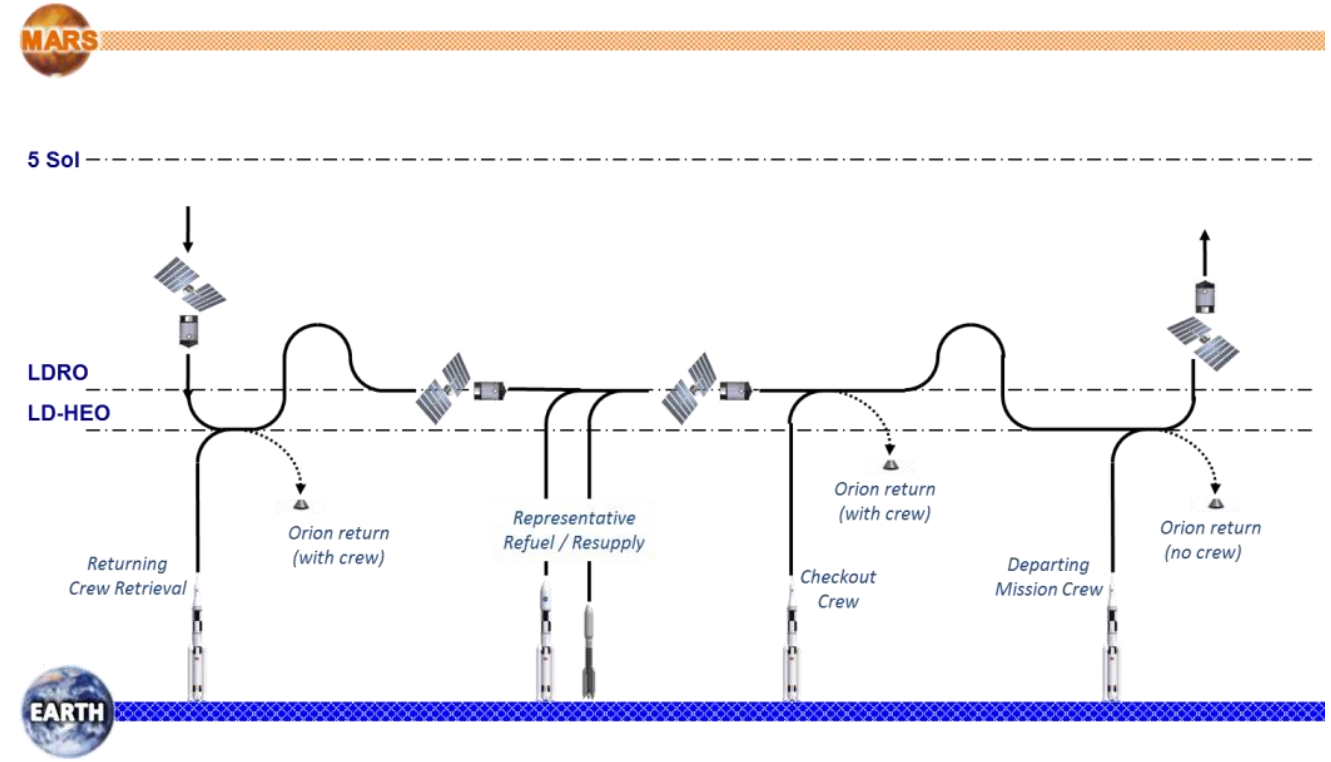

Figure 10. High-level depiction of mission con-ops for preparing crew transit stack for reuse.

\section{B. Discussion}

The major functions necessary to prepare the transportation stage for reuse, referring back to Figure 1, are propellant replenishment; refurbishment and repair, to include planned component replacement; addressing malfunctions and replacement of failed components; and functional verification. Additionally, some degree of safing and assembly and handling may be required depending on specific design and operations decisions. The study effort to date has been primarily focused on developing approaches to deal with propellant replenishment, as addressed in the following sections.

\section{Fuel Resupply}

For a propulsion stage that will be used for multiple missions, the most critical function is the ability to be resupplied with propellant and other operational fluids and gasses. Within the HPS architecture, two options are being

American Institute of Aeronautics and Astronautics 
traded for resupplying propellants: a refuel tanker and a dockable tank module. Both options have advantages and bring their own challenges.

\section{a) Refuel Tanker}

The refuel tanker is currently conceived of as a single-use spacecraft carrying propellant as payload that is able to transfer and maneuver itself from some initial Earth orbit to a rendezvous with the HPS in LDRO (Figure 11). The tanker concept assumes transfer of chemical propellants using pumps located on the hybrid stage and uses low-pressure, passive transfer of xenon and helium from tanker to hybrid stage, followed by compression on the hybrid stage. A refuel tanker represents a higher degree of reusability than the tank module option in that 1) it is closer to a "pump-and-go" resupply capability and 2) it potentially reduces the amount of disposed hardware (e.g., if the tanker can be reused).

Chemical refueling of both hydrazine and NTO has been demonstrated in space and is frequently performed on ISS by the Russians. Although the specific method of transfer to employ between the tanker and hybrid module may differ, there is high confidence that this will be a relatively straightforward operation to accomplish. However, the use of NTO and the negative effects it has on valves, seals, and other engine and propellant feed system components can impact the long-term reusability and lifetime of the propulsion system. Additional concerns include propellant toxicity and the potential for leakage and contamination, which provide a serious hazard to humans and could factor into

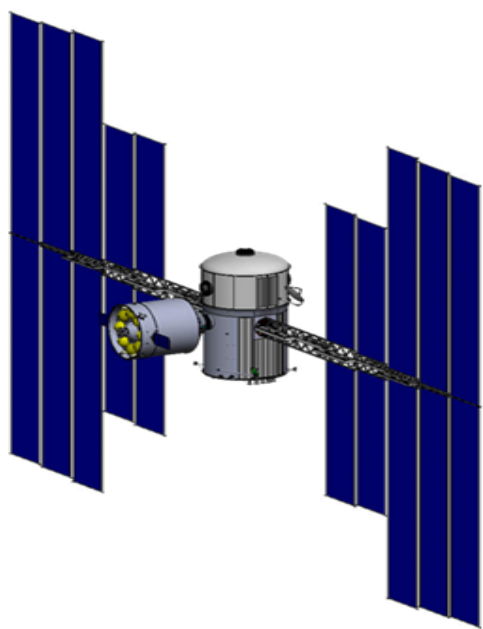

Figure 11. Refuel tanker servicing hybrid transit vehicle. servicing.

SEP xenon refuel in space is a capability in the early stages of development. Tight temperature and flow-rate tolerances that are imposed on transfer during ground servicing will also apply in space in order to maintain xenon in a supercritical state. This drives the time required to transfer the propellant and adds complexity to the system. Cryogenically-stored xenon may provide an alternative, but the cryogenic systems add complexity and mass, and additional developments would be required to enable the capability.

For both chemical and SEP systems, it will be infeasible to completely deplete the refuel tanker's supply tanks according to known methods, so there will be excess fluids that are not transferred. For pressure-fed transfer of storable chemical propellants using bellows, such as used for ISS, as well as for gasses this could be as much as $50 \%$ of the tanker capacity. This excess amount can be reduced by incorporating pumps and compressors, as on the current tanker concept. In order to avoid a high-pressure gas/fluid transfer interface between the tanker and transit stage, however, the pumps will need to be carried on the transit stage, adding mass and complexity, and introducing potential failure point that could affect the future servicing needs of the spacecraft.

In addition to propellants, pressurant must also be replenished. Pressurant refill is very challenging in space. In order to boost the pressure of one gaseous helium (GHe) tank into another, an elaborate compressor and thermal system will need to be designed, and maintenance of such system introduces additional complexity into refurbishment activities. Alternative solutions could eliminate the need for such complexity. For example, qualifying a GHe tank for the tanker to a much higher than normal pressure, perhaps 8000 or 10,000 psi and fill the client GHe tank through an orifice and isolation valve until the tank pressures are balanced. This will result in some GHe waste on the tanker side, but could enable a simpler transfer system and operation. Alternatively, the GHe tank on the hybrid vehicle could be oversized so that it contains sufficient pressurant to meet anticipated needs over the life of the vehicle. This can add considerably more mass and volume requirements to the hybrid vehicle, however.

Another hurdle will be refurbishment and repair of HPS modules designated to carry cargo and other uncrewed systems. Within the current architecture, crew are not available to facilitate servicing these systems, so a means must be provided to enable component replacement and other repair work. While robotics can provide the necessary manipulation capability, servicing systems will need to either operate autonomously or else be teleoperated by ground personnel from Earth. Autonomous servicing capabilities under development are beginning to be realized, but are still limited. Missions like Orbital Express demonstrated that it is possible to plan and execute autonomous replacement of some components, if the serviced spacecraft is designed to facilitate access to those parts. Identifying and planning for these operations in the development stage could lead to capabilities to directly address the challenges with remote servicing, and knowledge of other potential component access and replacement needs will inform the development of other robotic and autonomous capabilities. Teleoperation can supplement autonomous operations, although the time delay from Earth can make such operations challenging. Given currently available capabilities, the ability to remotely service uncrewed assets will be limited. Having crew available in the near term to perform or support servicing tasks

American Institute of Aeronautics and Astronautics 
opens up the possibility of performing much more extensive maintenance and the ability to address a wider variety of unplanned repairs and contingencies. Crew operations not already associated with a planned Mars mission may be quite expensive to conduct, however, and this may offset the advantages of reuse. The trade to balance crew operations, robotic operations, and degree of reuse is a trade that will need to be conducted.

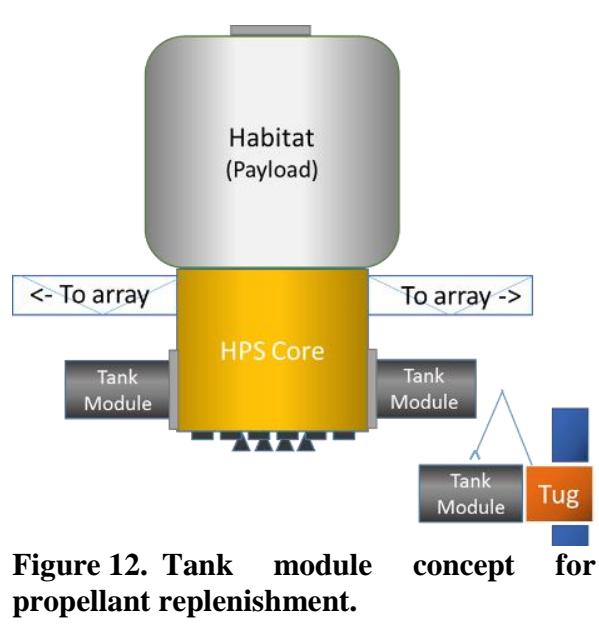

\section{b) Fuel Tank Module}

Replaceable, mountable tank modules are an alternative to refueling. Instead of transferring propellant from a tanker to the HPS propellant tanks, modules containing empty propellant tanks on the HPS are removed and replaced (Figure 12). Depending on design, one or more tank modules can be used. In the current concept, two modules are included. Each tank module is identical, and contains both SEP and chemical propellant tanks, pressurant tanks, an interface for mating with and connecting the propellant supply to the core vehicle, and may also include any limitedlife components designated for planned replacement. The core vehicle would house the reciprocal interface, accumulator tanks, the propellant feed system, and the thrusters. All major power system components would also reside on the core vehicle. A separable service module, or tug, transfers each tank module from launch through rendezvous and mating. Mating may occur via berthing or autonomous docking. If berthing, one of the vehicles will need to provide a robotic manipulator. The tug would also facilitate removal and disposal of expended modules. The modules could use the same mating interface as the refuel tanker, although some additional structural stabilization may be required to support the modules during transit, and a second interface would be needed if two modules are used to balance center of gravity, as with the current concept.

Because a substantial portion of the vehicle is replaced with each mission, thus breaking the functional integrity of the propellant system, the modular concept represents a lower degree of reusability than refueling. Consideration must be given to the multiple interface connections that would be required. Advancing capabilities can simplify these interfaces, but some degree of built in tolerance or compliance will likely be needed to facilitate the mechanical mating. Additionally, each fluid connection will need to be verified and leak checked following mating before operations commence.

Tank modules, however, are more attainable with current technologies. They do not depend on more advanced capabilities associated with refueling, and can overcome several of the challenges presented by using a refuel tanker, such as thermal management of the propellants during transfer. Furthermore, there is less likelihood of waste from nontransferable propellant. Once attached, the tank modules become part of the propellant feed system. Propellants and pressurant are transferred to the core stage through a regulated, low-pressure interface, where they are collected into accumulator tanks, which then feed the engines.

Tank modules also provide a ready solution to planned replacement or upgrading of life-limited components. Components are pre-integrated and functionally verified on the ground and interface with the core vehicle through a relatively simple connection, thereby maintaining a higher level of functional integrity than if removal and replacement was required. Furthermore, the core system does not need to be designed for accessibility in limited real estate, or need to provide a capability to facilitate swapping out the items telerobotically or autonomously.

As currently conceptualized, the tank module concept will still have the same life-limiting concerns associated with the negative effects of NTO as the refuel tanker option for those parts of the system residing on the core module. Furthermore, while planned replacement of components is simplified with the modular approach, a means to deal with unanticipated failures and maintenance on the core stage must still be provided. A more thorough assessment of the ability to address these contingencies through redundancy or allowable degradation of capabilities, and an assessment of the availability of greater servicing capabilities in line with recent servicing demonstrations has yet to be performed.

Despite the lesser degree of reusability initially attained, the lower apparent development needs for incorporating tank modules into the HPS architecture can lead to greater degrees of reusability over time. Designing in a capability for the tug to draw propellant from the tank modules could facilitate a more fully reusable architecture by extending the operational life of the tug, thus allowing it to return expended modules and rendezvous with additional logistics or tanker modules for delivery to the transit stack. If tank modules could be returned to Earth or to space-based infrastructure for refurbishment and refueling, they could also potentially see reuse for multiple missions.

American Institute of Aeronautics and Astronautics 


\section{High-Power Solar-Electric System}

In addition to the propulsion systems, the high-power solar-electric system on the hybrid module provides a significant potential to affect the reusability of the system. Although the ability to refuel the propulsion stage is critical for mission-to-mission reuse, the most critical driver for HPS lifetime given the current design may be the solar arrays. The power derived from the solar arrays directly impacts travel times to Mars. In order to maintain reasonable trip times to Mars for the crew the arrays must provide a minimum level of power, although cargo missions can still be performed at lower power levels. Since solar arrays degrade with exposure to the space environment, the array design must be sized large enough at beginning of life to retain sufficient power at the end of life. The mass associated with larger arrays, including the mass of the power management and distribution system needed to handle the maximum power provided by those arrays, can increase quickly as power levels increase, so a balance must be struck between the BOL power provided and the serviceable years that the system can provide. Furthermore, because of the size of the array wings and inherent danger of operating near high-power arrays, which can develop and carry a very large differential charge, the ability to service or replace array wings or associated components may be limited and very risky.

Developments that can protect the arrays and reduce the rate of degradation will enable longer lifetimes for highpowered solar systems. Another alternative to provide longer-life EP systems would be to replace solar power with nuclear power. Nuclear propulsion technologies are in development but may not be available for earlier missions. However, they do provide an option for when the desired system lifetime of high-powered systems can no longer be efficiently achieved with solar arrays.

Other power system components also present design considerations and could impact servicing and reusability of the system. Space Station experience with the Solar Array Rotary Joints has demonstrated that the long-term wear of mechanical components must be considered, and that a plan for periodic maintenance may be required. Also, consideration must be given to accessibility of components such as the power management and distribution systems and power processing units. These components typically have multiple connections, which complicates any replacement operations, especially if the parts are not easily accessible. With the collection of radiators, tanks, and fluid lines, it is all too possible for these components to become buried in the structure and be inaccessible for direct servicing unless their placement is consciously considered during the design phase or else sufficient redundancy is incorporated into the design to eliminate the need for servicing.

\section{Other Considerations}

Regardless of approach, the initial ability to repair the spacecraft will be limited by the ability to provide the right parts at the right time. Because of the distance from Earth to where servicing will occur and the time required to deliver repair parts, it will be critical to have early awareness of those components that may require replacement or repair. With current launch rates, even fast transits to rendezvous with the spacecraft will require some predictive capability to ensure the correct parts are on hand. Increasing use of integrated health monitoring and making use of flight or digital twin data can facilitate with predicting potential failures and planning for the servicing mission. In time, as the ability to stockpile or manufacture spare parts in space is developed, greater flexibility and responsiveness to repair needs can be achieved. Increasing launch availability and reducing payload preparation time on the ground, and continued development of in-space manufacturing technologies such as 3D printing, could enable a more rapid response capability to more readily address unanticipated repairs.

Between lifetime limitations due to degradation and the pace of development that leads to the potential to upgrade capabilities over time; avionics, computers, other electronics, and batteries are primary candidates for planned replacement strategies. Incorporation of a standard interface would facilitate such a strategy by ensuring that new modules will readily fit into the system, if they are not replaced as part of a tank module. A further consideration, particularly with electronics and software, would be to make sure that the functionality of the new modules is compatible with, and does not interfere with, existing components.

Structures will need to be robust to the longer durations in space, particularly if they will spend appreciable amounts of time in regions with high concentrations of micro-meteoroids and orbital debris. Current approaches for protection include adding shielding and overdesigning structures, both of which can add substantial mass as flight durations increase. Integrated structural health monitoring and self-healing capabilities can improve damage detection and tolerance, and reduce the additional mass required, which will facilitate longer lifetimes for system structures.

Other subsystems have not been assessed at this time, but they will benefit from similar means to monitor and predict degraded performance and failure, and will have to make similar decisions regarding incorporating redundancy, planned replacement, and accessibility.

American Institute of Aeronautics and Astronautics 


\section{Making a Reusable Architecture a Reality}

It will take time to advance from the current paradigm of expendable systems to realizing the vision for reusability. New capabilities and technologies must be developed, demonstrated, and built upon to move beyond currently accepted operations. Without a systematic approach to development, campaigns will likely be limited to expendable sorties for some time.

A logical, systematic approach to realizing a fully-reusable interplanetary transportation system begins by demonstrating critical capabilities needed by EMC systems, such as high-power SEP in the proving ground. Operating those demonstration systems will provide insight into system behavior and drive out points of failure that could impact reuse. Use the lessons learned to develop technologies and capabilities that increase reusability and serviceability, taking advantage of rapid development cycles to explore multiple options when possible, and incorporate the new capabilities into EMC system designs.

Demonstrate the new capabilities and evaluate their performance through realistic operations in the proving ground, first on available systems such as initial cis-lunar habitation and transportation systems, and then with the first generation units of EMC mission vehicles. While designing for reusability, limit planned use of the first units of new EMC systems to a single mission that verifies the systems and servicing capabilities incorporated into the design. Identify modifications to better enable reusability in later system builds and future designs. Use of the proving ground should not cease once full operations on Mars have begun. Rather, the proving ground should continue to be used to flight test and evaluate new capabilities prior to deployment far from the Earth. Additionally, repurpose left-over hardware after servicing missions to build up infrastructure to support future missions and new opportunities and to provide platforms for demonstrating emerging capabilities as they are developed.

Realizing a reusable architecture will take more than just a plan for developing and testing capabilities. The general mindset must also shift towards designing for reuse. Traditional design attempts to optimize for a primary mission of a certain duration, and makes certain design decisions as a result. As described in this paper, when designing for reuse, other factors influence design choices and different capabilities may be required. In order to facilitate this shift in mindset, the availability of inexpensive, rapid development methods are needed to more readily investigate promising new capability options.

A shift is also needed towards more effectively leveraging the ingenuity, development capabilities, and competitive spirit of industry, academia, and international partners for developing the necessary capabilities to enable fully reusable architectures. By purchasing available capabilities, NASA provides an initial customer base that promotes a stronger economic motive for advancing pioneering efforts in space, and frees itself to focus internal development efforts on meeting the challenges of deep-space human spaceflight.

In order to fully realize the benefits of reusability, the synergy between utilization of space resources and reusability must be understood and leveraged. The idea of utilizing space resources truly shows its value when combined with reusable systems and reusable infrastructure, and to obtain the maximum benefits from reusability, utilization of space resources is necessary.

\section{Conclusion}

Although implementation of the vision of full reusability in space is not immediately possible, capabilities are currently available that enable designers to develop systems with a limited degree of reusability that will provide experience and knowledge on the path to the vision. Major challenges exist that will impact the ability to service and prepare systems for reuse, such as propellant refuel and the ability to remotely service uncrewed vehicles, particularly when dealing with unplanned repairs that exceed current autonomous or robotic capabilities. However, these challenges are currently being addressed by several ongoing development efforts, and several initial capabilities have already been demonstrated in space by both humans and robots. While incorporating higher levels of reusability is not a viable place to begin, available alternatives that have lower degrees of reusability can still benefit the architecture and need to remain in the decision space as they may be less risky or costly.

Incorporating these existing capabilities into an EMC architecture lays a foundation for reusability that can be built upon by using the cis-lunar proving ground to evaluate the performance and reliability of reusable systems and demonstrate new capabilities to support reusability. As capabilities and experience grow through mission use and further proving ground demonstrations, greater degrees of reusability will be attainable and new opportunities for incorporating reusable systems and infrastructure will become available.

\section{Acknowledgments}

The team would like to recognize the important contributions of the SMEs who shared their wealth of knowledge and experience with spacecraft systems, development of servicing capabilities, and existing challenges with system

American Institute of Aeronautics and Astronautics 
longevity and reuse. The SMEs that supported this investigation into incorporating reusability into spaceflight architectures are: John Brophy, Kurt Hack, David Manzella, Hoppy Price, Melissa McGuire, Patrick McRight, and Marius Popescu,

\section{References}

${ }^{1}$ Williams, G., and Crusan, J., "Pioneering Space - the Evolvable Mars Campaign," presentation to the NASA Advisory Council, Washington, D.C., April, 2015.

${ }^{2}$ Goodliff, K., Cirillo, W., Mattfeld, B., Stromgren, C., and Shyface, H., "Comparison of Human Exploration Architecture and Campaign Approaches," AIAA Space Conference and Exposition, Pasadena, CA, 2015 (submitted for publication).

${ }^{3}$ Merrill, R., Chai, P., Jones, C., Komar, D.R., and Qu, M., “An Integrated Hybrid Transportation Architecture for Human Mars Exploration," AIAA Space Conference and Exposition, Pasadena, CA, 2015 (submitted for publication).

4 Toups, L, et al., "Pioneering Objectives and Activities on the Surface of Mars," AIAA Space Conference and Exposition, Pasadena, CA, 2015 (submitted for publication).

5 "Concluding Remarks by Dr. Wernher von Braun about Mode Selection for Lunar Landing Program," 7 June 1962, Lunar-Orbit Rendezvous File, NASA Historical Reference Collection, NASA Headquarters, Washington, D.C.

${ }^{6}$ Miller, C., et. Al., "Economic Assessment and Systems Analysis of an Evolvable Lunar Architecture that Leverages Commercial Space Capabilities and Public-Private-Partnerships," NextGen Space LLC Report, 2015

7 Wilhite, A. and Chai, P., "Plan B for U.S. Human Space Exploration Program," AIAA Space Conference and Exposition, San Diego, CA, 2014.

8 Griffin, J.W., "Background and Programmatic Approach for the Development of Orbital Fluid Resupply Tankers," AIAA/ASME/SAE/ASEE Joint Propulsion Conference, AIAA-86-1601, Huntsville, Al, 1986.

9 Dipprey, N. F., and Rotenberger, S. J., "Orbital Express Propellant Resupply Servicing," AIAA/ASME/SAE/ASEE Joint Propulsion Conference and Exhibit, AIAA 2003-4898, Huntsville, AL, 2003.

${ }^{10}$ Flight Production Generic Templates," JSC 25187, Appendix A., NASA Johnson Space Center, Houston, TX.

${ }^{11}$ McCleskey, C. M., "Space Shuttle Operations and Infrastructure: A Systems Analysis of Design Root Causes and Operator Needs with Implications for Exploration Systems Development," Presentation charts 40-41, NASA Kennedy Space Center, October 2005.

${ }^{12}$ Fredrickson, S. E., Lockhart, P. S., and Wagenknecht, J. D., "Autonomous Extravehicular Robotic Camera (AERCam) for Remote Viewing," Proceedings of the AIAA International Space Station Service Vehicles Conference, Houston, Texas, 1999.

${ }^{13}$ Ahlstrom, T. D., et al., "Robonaut 2 on the International Space Station: Status Update and Preparations for IVA Mobility," AIAA Space Conference and Exposition, AIAA 2013-5340, San Dieago, CA, 2013.

14 "Servicing History and Long-Term Plans", NASA Facts, NF-195, 1993.

${ }^{15}$ Adams, T. A., Rhodes, R. E., "A Process for Developing and Balancing Quantitative Safety, Reliability, and Maintainability Requirements," KSC Reliability Website: http://kscsma.ksc.nasa.gov/Reliability/Documents/SRMrequire.pdf

${ }^{16}$ Space Shuttle Program's "Requirements and Procedures for Certification of Flight Readiness," NSTS 08117

Rev. M.

17 “STS-120 (23 ${ }^{\text {rd }}$ Space Station Flight) Discovery", NASA Facts, FS-2007-12-033-KSC, 2007.

${ }^{18}$ Chai, P., Merrill, R., and Qu, M., "Mars Hybrid Propulsion System Trajectory Analysis Part I: Crew Missions," AIAA Space Conference and Exposition, Pasadena, CA, 2015 (submitted for publication).

${ }^{19}$ Merrill, R., Strange, N., Qu, M., and Hatten, N., "Mars Conjunction Crewed Missions with a Reusable Hybrid Architecture," IEEE Aerospace Conference, Big Sky, MT, 2015.

${ }^{20}$ Strange, N., et al., "Overview of Mission Design for NASA Asteroid Redirect Robotic Mission Concept," 33rd International Electric Propulsion Conference, The George Washington University, Washington, D.C., October 2013.

${ }^{21}$ Chai, Merrill, and Qu, "Mars Hybrid Propulsion System Trajectory Analysis Part 1."

${ }^{22}$ Chai, P., Merrill, R., and Qu, M., "Mars Hybrid Propulsion System Trajectory Analysis Part II: Cargo Missions,” AIAA Space Conference and Exposition, Pasadena, CA, 2015 (submitted for publication).

${ }^{23}$ Qu, M., Merrill, R., Chai, P., and Komar, D. R., "Trajectory Designs for a Mars Hybrid Transportation Architecture,” AAS/AIAA Astrodynamics Specialist, Vail, CO, 2015 (submitted for publication).

American Institute of Aeronautics and Astronautics 